\title{
Self-Termination/Anergic Mechanisms in Human Basophils and Mast Cells
}

Donald W. MacGlashan, Jr. Johns Hopkins Asthma and Allergy Center, Baltimore, Md., USA

\section{Key Words}

Basophil activation $\cdot \mathrm{Fc}$ receptor (high-affinity $\lg \mathrm{E}$ receptor) - Basophils, human - Mast cell, human ·

Regulation $\cdot$ Signal transduction $\cdot$ Anergy $\cdot$ Desensitization in itself, an enormous topic and it should be said that in order for a cell to return to a resting state of activation, there must be many layers of homeostatic mechanisms. For illustrative purposes, consider the simple model of receptor-mediated activation shown in figure 1 . In the simplest scheme, figure 1a shows only the activation events that occur early in the reaction. In this simple scheme, each step involves a kinase that phosphorylates its substrate, which is also a kinase of some kind. In order for these steps to be controlled, two feedback loops are introduced into the model. In figure 1 , positive or activating steps are designated as P1, P2, etc., and negative or deactivating steps are designated as $\mathrm{N} 1, \mathrm{~N} 2$ and so forth. But note that in this model, the negative components are activated by positive components. It does not have to be this way, but experimental data suggest that this is a common way of activating or recruiting negative components. For the cell to settle back to the resting state, there must be another layer, shown in figure 1c. These components (designated $\mathrm{CN} 1$, etc.) could also be activated, which would necessitate another layer or they could be constitutively active. To return to the original resting state, it seems likely that something must stop the original signal. In other words, although the various persistent negative feedback mechanisms could terminate an ongoing reaction, by themselves they could not return the system to its resting state.
To understand deactivation or self-terminating signaling, it is first necessary to explore the steps that occur during activation that lead to cellular functions. This is,

\section{KARGER}

Fax +41613061234 E-Mail karger@karger.ch www.karger.com (c) 2009 S. Karger AG, Basel

$1018-2438 / 09 / 1502-0109 \$ 26.00 / 0$

Accessible online at:

www.karger.com/iaa
Correspondence to: Dr. Donald W. MacGlashan, Jr.

Asthma and Allergy Center

Johns Hopkins University

5501 Hopkins Bayview Circle, Baltimore, MD 21224 (USA)

Tel. +1 410550 2130, Fax +1 410550 2145, E-Mail dmacglas@jhmi.edu 


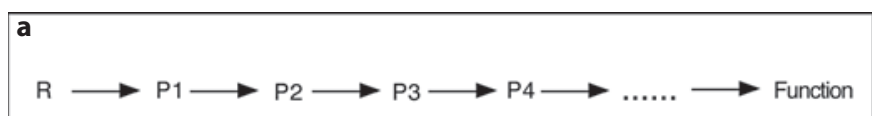

b

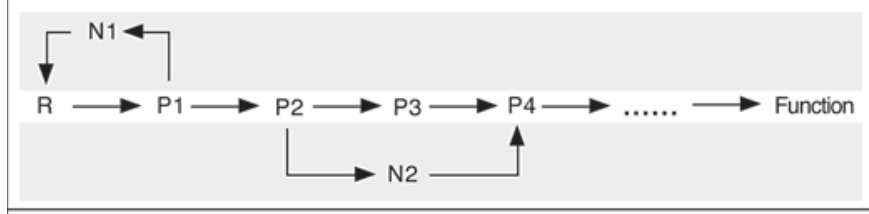

C

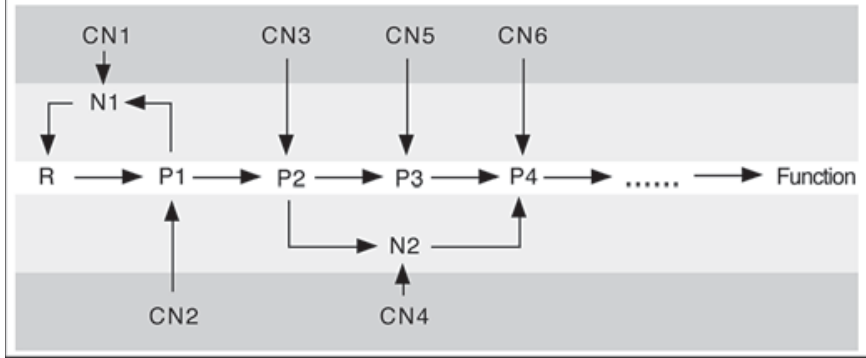

d

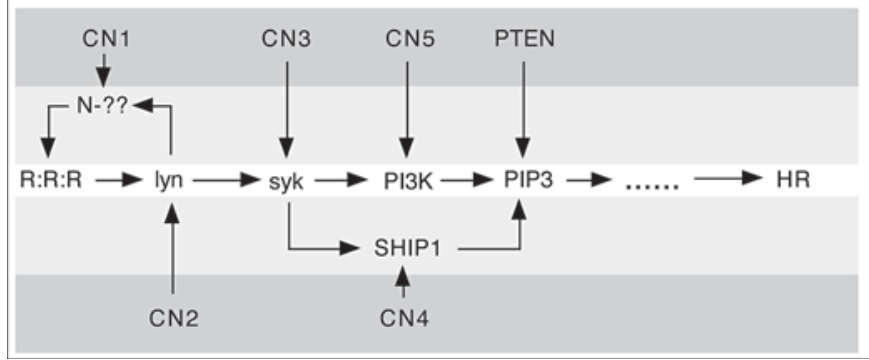

Fig. 1. Simplified schematics of early signaling; activating and deactivating mechanisms. a Activation cascade as a sequence of kinase reactions. $\mathbf{b}$ Overlay of negative feedback loops on the activation cascade. P1, P2, etc., are activating elements, N1, N2, etc., are negative (deactivating) elements. c Second layer of homeostatic enzymes. In this simple scheme, the next layer consists of phosphatases that are constitutively active (CN1, CN2, etc.). d Substitution of some of the abstract elements in $\mathbf{c}$ with elements known to participate in IgE-mediated signaling $(\mathrm{R}=\mathrm{Fc} \varepsilon \mathrm{RI}$ and $\mathrm{HR}=$ histamine release). Only one constitutively active element has been identified with some assurance, PTEN. There are several other known phosphatases that have been identified in mast cells, but their targets and roles remain unknown. It seems likely that the 'CN' phosphatases are represented by a much smaller number than indicated here (where there is one phosphatase for each signaling element); for example, it may be that $\mathrm{CN} 1=\mathrm{CN} 2=\mathrm{CN} 4$ and $\mathrm{CN} 2=\mathrm{CN} 5$.

\section{IgE-Mediated Signaling}

With this abstract basis as a background, the IgE-mediated reaction can be overlaid. In figure $1 \mathrm{~d}$, a few of the pieces have been applied. The reaction starts with aggre- gation of the IgE receptor. Numerous studies have demonstrated that both activation and deactivation events require the continued presence of aggregates. Dissociation of the aggregates results in a rapid return of all signaling components, positive and negative, to their resting state, with the provision that this statement only applies to an early time frame. There are deactivation events that cannot be reversed if sufficient time passes, generating a form of activation memory. The details of what events aggregation induces are still to be discovered, but it seems clear that one early step is the association and activation of a src family kinase. For murine mast cells, lyn, fyn and hck have been implicated [1-7], but the canonical first step is the activation of lyn kinase. Lyn phosphorylates the ITAM of FceRI $\beta$ and $\gamma$ leading to the recruitment and activity of syk (spleen tyrosine kinase). At this point, there are many branching steps. Lyn, fyn and syk mediate the phosphorylation of many proteins that carry the reaction forward. One of these steps, mediated by syk, is the phosphorylation of the PI3 kinase regulatory subunit, p $85 \alpha$, which results in recruitment of PI3 kinase and its activation. This enzyme has a primary target, the phosphorylation of phosphatidyl 4,5-bisphosphate in the membrane, resulting in the generation of phosphatidylinositol-3,4,5-trisphosphate (PIP3). PIP3 acts as a recruitment site for a variety of proteins (PLC $\gamma$, btk, PDK1/2) which also carry the reaction forward. In human basophils, PI3 kinase is absolutely required for secretion of all 3 classes of mediators $[8,9]$ (prestored mediators like histamine, newly synthesized, rapidly released mediators like LTC4 and newly synthesized, slowly released mediators like IL-4), suggesting that the generation of PIP3 is a critical second messenger. Stopping at this point, it is possible to add two downregulatory steps. The first is an early feedback loop that modulates the activity of the earliest steps. This step will be explored below, but is currently unknown. The second feedback step to add is the recruitment of the lipid phosphatase, SHIP (SH2-containing inositol phosphatase).

In human basophils, it is possible to use a methodological maneuver to demonstrate the engagement of deactivation mechanisms. First, a simple inspection of the kinetics of histamine release demonstrates the presence of deactivation. Only rarely can human basophils be induced, under natural conditions, to release all of the preformed granule contents, i.e. $100 \%$ histamine release. It is not because the granules cannot be secreted, but that there is a self-limiting process that stops the release process and this can be demonstrated by several methods [10]. This self-limiting process can be demonstrated in 


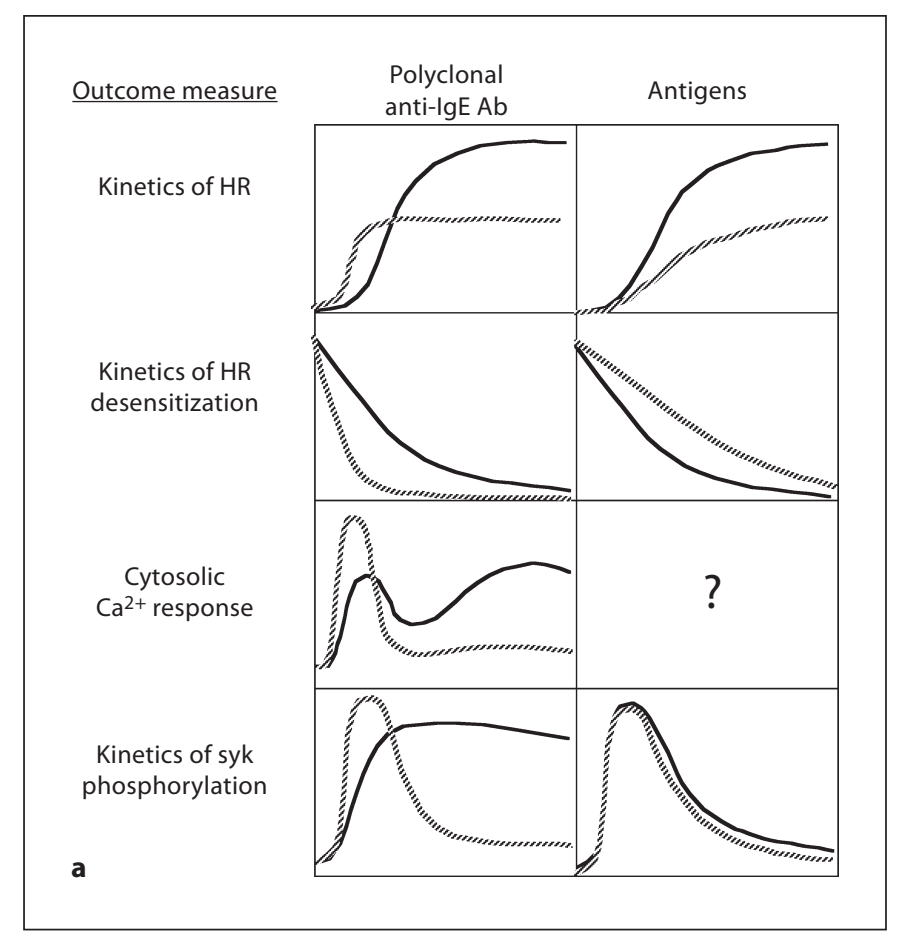

Fig. 2. Differences in the basophil response to polyclonal anti-IgE antibody vs. antigens (or monoclonal anti-IgE antibodies). The data are derived from several previously published $[15,25]$ and unpublished studies. a Kinetics of four outcomes following stimulation with either a concentration of stimulus that is optimal for histamine release (black line) or supraoptimal (hatched gray line) is shown. $\mathbf{b}$ Phosphorylation of SHIP on the supraoptimal side of a dose-response curve for histamine release relative to the response to an optimal concentration of the stimulus. Purified human basophils were stimlulated with either goat polyclonal anti-

another way. Stimulation of the basophil in the absence of extracellular calcium results in no mediator secretion [11]. If this condition is maintained for $45-60 \mathrm{~min}$ and calcium is returned to the reaction, no secretion occurs (compared to simply incubating the cells in the absence of extracellular calcium). This operational method has been termed desensitization. The decay of the cell's ability to secrete during the $45 \mathrm{~min}$ has the general appearance of a first-order decay process (e.g., see fig. 2, desensitization panels). Depending on the strength of the signal during the calcium-free phase of the experiment, the basophil's response to non-cross-reacting antigens may also be partially blunted $[12,13]$. The desensitization to the aggregating antigen is termed specific desensitization and the blunting of the response to other antigens is called nonspecific desensitization. Notably, these two forms of desensitization refer only to IgE-mediated acti-

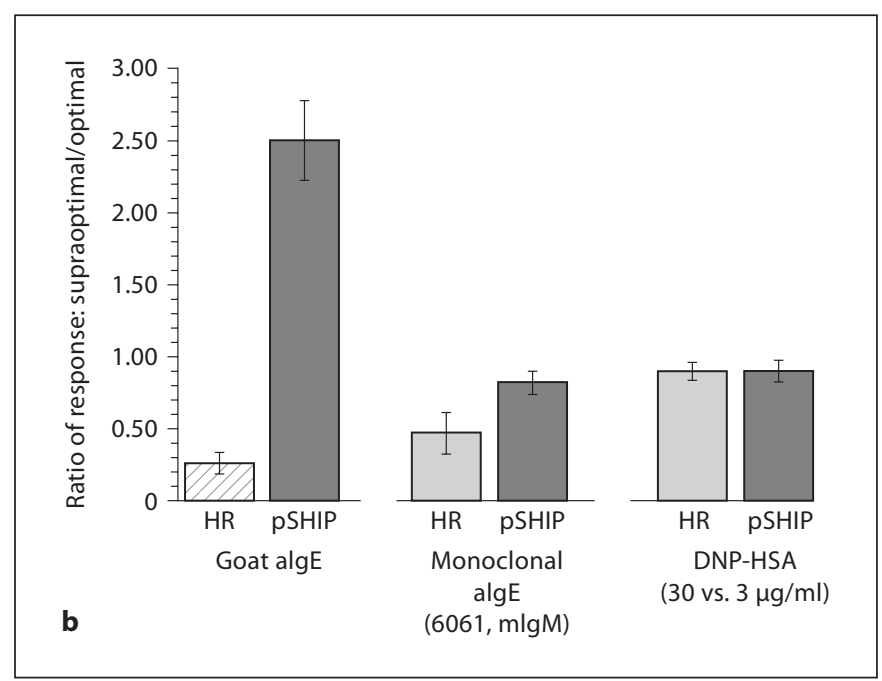

IgE antibody or monoclonal IgM anti-hIgE antibody (6061) at a concentration considered optimal or supraoptimal for histamine release. The ratio of the supraoptimal to optimal response is plotted for histamine release or phosphorylation of SHIP-1 (as detected on a Western blot). In a similar design, basophils were sensitized with DNP (dinitrophenyl)-specific IgE and stimulated with an optimal or supraoptimal concentration of DNP-HSA. $\mathrm{HR}=$ Histamine release; $\operatorname{aIgE}=$ anti-IgE; $\operatorname{mIgM}=$ monoclonal IgM.

vation. Activation through other types of receptors is usually intact [10], although one study found some IgEmediated blunting of an FMLP-induced (G-proteinlinked receptor) response [14] if the basophils were treated with wortmannin. But by and large, most experiments suggest that the processes of downregulation that occur apply only to signaling cascades that have relevance to IgE-mediated secretion.

\section{Signaling and Self-Termination}

The relationship of signaling steps to these operationally defined forms of desensitization is an area of active investigation. For reasons not yet understood, stimulating human basophils with polyclonal anti-IgE antibody results in a sustained activation of signaling elements like 
syk and its substrates for at least 1-3 h [15]. For example, the activity of PI3 kinase is sustained. In contrast, stimulation with antigens (for basophils sensitized with the appropriate antigen-specific IgE) results in transient activation of all early signaling molecules. Looking somewhat downstream of PI3 kinase, anti-IgE antibody induces only a transient activation of events dependent on PIP3. In human basophils, both phosphorylation of Akt and activation of the entire p21ras to Erk pathway are transient, with their respective phosphorylation states returning to near resting levels by $60 \mathrm{~min} \mathrm{[10].} \mathrm{Therefore,}$ this stimulus highlights a point in the signaling cascade that is experiencing downregulation; some process results in the metabolism of PIP3. If basophils are desensitized with antigen (i.e., they are stimulated under calcium-free conditions), and restimulated in the presence of extracellular calcium and a non-cross-reacting antigen (after dissociating the first antigen), the early signaling steps are intact, but the steps downstream of PI3 kinase are blunted [10]. These two pieces of information lead to a proposal that nonspecific desensitization is operative at the point of PIP3 metabolism. The current hypothesis is that one of the SHIP proteins is responsible for the metabolism of PIP3 at this point in the reaction. What PI3 kinase generates, SHIP takes away. With sufficient recruitment of SHIP, the reaction cannot penetrate the required generation of PIP3, i.e. the reaction can be fully active upstream of PIP3, but SHIP provides a barrier to downstream activation.

In basophils and mast cells, SHIP1 has been shown to translocate to the plasma membrane during stimulation [16]. PTEN, a phosphatase that is also capable of metabolizing PIP3, is not dynamically activated during stimulation. It is present and probably acts to establish a set point for activating the cells, but SHIP is dynamically recruited to the reaction complex. There are no studies that demonstrate a way to increase the intrinsic enzymatic activity of SHIP, but during stimulation, SHIP is phosphorylated and this phosphorylation site allows SHIP to be recruited to the activation complex [17-19]. In other words, SHIP is one of the dynamically activated negative signaling elements. Studies in SHIP-/- mice demonstrate the remarkable impact of not having SHIP available to control this reaction [20]. Mast cells become exquisitely sensitive to IgE-mediated stimulation. There are pathological states in humans that appear to result in basophils with lower levels of SHIP expression and result in basophils that are more sensitive to stimulation [21]. There are also instances where SHIP is overexpressed and the IgEmediated response is blunted [22].
Studies of the supraoptimal side of the dose response curve for anti-IgE antibody in basophils show that SHIP is more heavily phosphorylated than at optimal levels of secretion [23]. These results suggest that for this stimulus, overrecruitment of SHIP prematurely shuts down secretion. It should be noted that this is a special property of stimulation with polyclonal anti-IgE antibody. Figure 2 summarizes some of the observations regarding the difference between a polyclonal anti-IgE antibody and antigens $[24,25]$. By and large, events happen faster but the response dampens more rapidly on the supraoptimal side of the dose-response curve for polyclonal anti-IgE antibody while for antigens, events are slower. The results for antigens are consistent with cross-linking theories for antibody-antigen complexes where supraoptimal antigen concentrations result in less or smaller aggregates than optimal concentrations and therefore should have functional consequences similar to events on the suboptimal side of the dose-response curve [26,27]. The speculation is that for polyclonal anti-IgE antibody, the optimum for histamine release is no longer the optimum for the number and quality of aggregates, as it is for antigen stimulation. Instead, the anti-IgE antibody generates even larger and more effective aggregates as one slides onto the supraoptimal side of the dose-response curve, but downregulatory effects begin to take precedence and mediator secretion suffers despite the presence of more and larger aggregates [24]. Consistent with these earlier observations, on the supraoptimal side of an antigen dose-response curve or a monoclonal anti-IgE antibody curve, SHIP phosphorylation is less than observed at the optimum (fig. 2), but as noted in figure 2 and in studies by Gibbs et al. [23], supraoptimal polyclonal anti-IgE antibody generates stronger SHIP phosphorylation.

It should be noted that although a role for SHIP in nonspecific desensitization has been discussed above, this same mechanism will contribute to specific desensitization as well. Indeed, this process is most obviously responsible for modulating the reaction complex forming with the aggregated receptor. How it escapes this reaction complex to participate in other future reaction complexes is not understood, but there are indications from studies in B cells that a complex of SHIP and dok $2 / 3$ may escape their initial reaction complex to modulate other forming reaction complexes, such as those initiated by a non-cross-reacting antigen.

SHIP is a family of phosphatases [28]. SHIP-1 is the best studied of the family although recent studies have examined the role of SHIP-2 in murine mast cell secretion [29]. In human basophils, only SHIP-1 appears to 


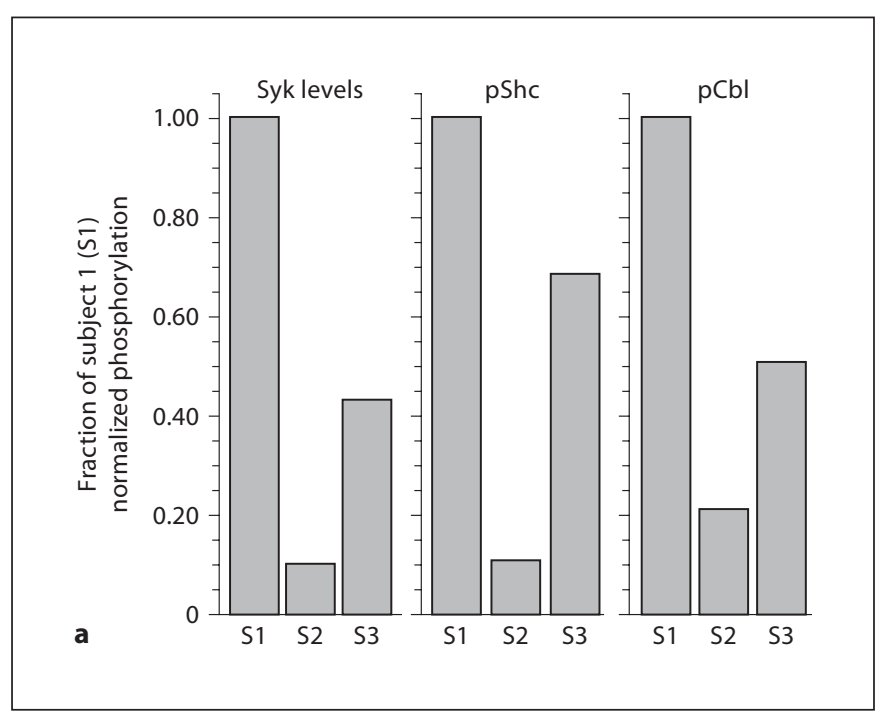

Fig. 3. The relative expression of syk is concordant with phosphorylation of its downstream substrates or histamine release. The data are derived from previously published studies [35]. a Basophils from 3 subjects were examined for their expression of syk or their response to stimulation with anti-IgE antibody (the bars represent the time average of the signaling element response in the first $15 \mathrm{~min}$ of the reaction; $\mathrm{pShc}$ and $\mathrm{pCbl}=$ phosphorylated forms of Shc and c-Cbl). b Inhibition of phosphorylation of syk substrates following IgE-mediated downregulation of syk. Purified human basophils were sensitized with DNP (dinitrophenyl)specific IgE and gp120 (HIV protein)-OVA (ovalbumin)-specific IgE. They were stimulated for $18 \mathrm{~h}$ with DNP-HSA in order to downregulate syk expression. In parallel, cells were incubated for $18 \mathrm{~h}$ without stimulation. After the 18 -hour incubation, both

translocate to the plasma membrane during activation [16], but there are indications that the expression level of SHIP-2 is different in some patients with chronic urticaria [22]. In basophils, phosphorylation of SHIP-1 appears to be dependent on src family kinase activity, not the activity of syk [16]. Therefore, one of the deactivation feedback loops thought to regulate basophil secretion does not depend on the activity of syk, but depends on the earliest known kinases of the reaction. In this context, desensitization of human basophils does not occur in the presence of a src family kinase inhibitor [30]. This is the first of several observations that desensitization depends not on the activities of syk but the activities of src family kinase members.

Self-Termination/Anergic Mechanisms in Human Basophils and Mast Cells

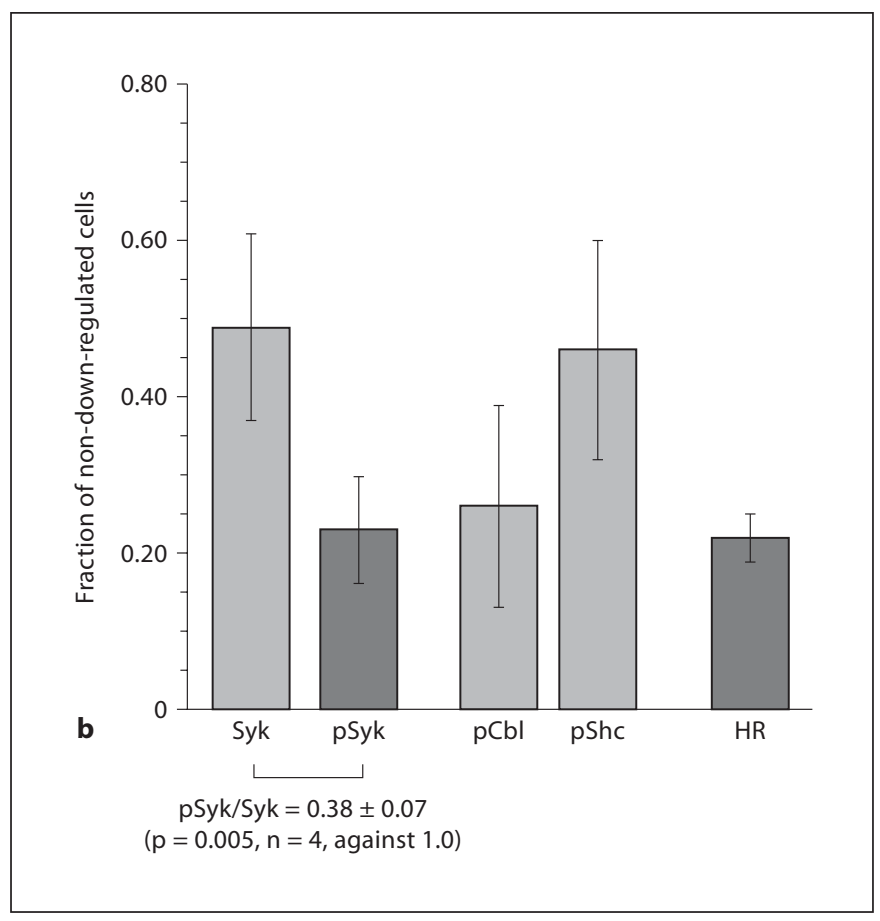

groups of cells were restimulated with gp120-OVA. Following 15 min of stimulation with gp120-OVA, the supernatants were harvested for the measurement of histamine release and cell pellets were lysed to either assess (1) the level of syk expression, (2) the level of c-cbl phosphorylation or shc phosphoylation (both putative substrates of syk), (3) the level of syk phosphorylation (using immunoprecipitation to capture syk) or (4) histamine release. The relative level of each outcome is plotted as the ratio to the cells that were not stimulated for $18 \mathrm{~h}$. Also shown is the ratio of the change in syk phosphorylation to syk. All outcomes were statistically different than no change (a ratio on the ordinate of 1.0). In addition, the decrease of syk phosphorylation was statistically lower than the decrease in syk expression (left two columns). HR = Histamine release.

As noted above, nonspecific desensitization appears to depend on the activity of SHIP-1, but there are other mechanisms that modulate nonspecific desensitization. The one that probably accounts for the majority of the effect late in the reaction is the loss of syk. Studies in human and rodent basophils and mast cells have shown that IgEmediated activation results in the loss of syk by the process of ubiquinylation and $26 \mathrm{~S}$ proteasomal degradation [31-34]. In human basophils, this mechanism operates slowly, at least relative to the self-terminating steps that appear responsible for stopping mediator secretion. Syk loss takes 1-8 h, self-termination steps operate in a time frame of minutes. SHIP is phosphorylated and recruited in minutes, but this state returns to resting levels within

Int Arch Allergy Immunol 2009;150:109-121 

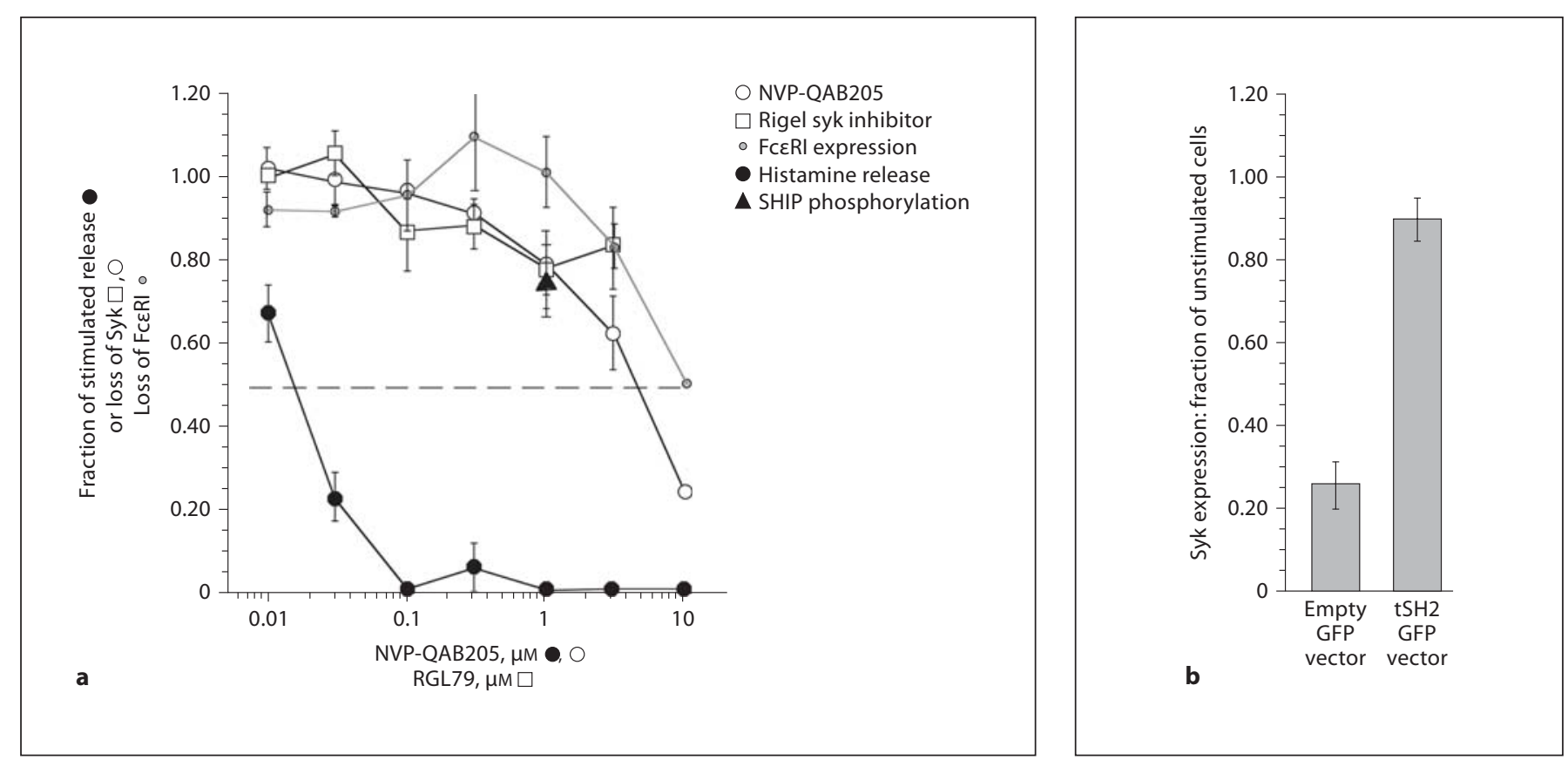

Fig. 4. Independence of downregulatory mechanisms from the activity of syk (abstracted from references $[35,42]$ and related but unpublished studies). a Purified human basophils were stimulated in the presence or absence of NVP-QAB205, a Novartis syk inhibitor with relative selectivity for syk [16], or a Rigel syk inhibitor, RGL79 [63], for $18 \mathrm{~h}$. The cell pellets were lysed and analyzed by Western blotting for the expression of syk, expression of FceRI or histamine release. The ordinate is the relative expression of syk or FceRI as compared to the loss that occurred following stimulation without the syk inhibitor. The dashed line is the point of $50 \%$ inhibition of the outcome. Also indicated by the filled triangle is the relative absence of SHIP phosphorylation inhibition. b Purified human basophils were transfected with either an empty GFP vector or a GFP construct linked to the tandem SH2 domains of syk (tSH2) [64]. After overnight culture to allow expression, the cells were stimulated for an additional $18 \mathrm{~h}$ with or without an optimal concentration of anti-IgE antibody to downregulate syk expression. The cells were analyzed by flow cytometry [65] for the presence of syk and coexpression of GFP. The plot shows the relative loss of syk in cells that were expressing GFP in each group. Presence of the tSH2 reversed the loss of syk induced by overnight stimulation with anti-IgE antibody. hours if the stimulus is anti-IgE antibody and $1-2 \mathrm{~h}$ if the stimulus is antigen. Nevertheless, after $2-3 \mathrm{~h}$, basophils continue to display nonspecific desensitization. The data support a model where the early stage of the nonspecific desensitization effect is mediated by SHIP and the later stage is mediated by loss of syk [16]. Studies of downstream signaling after loss of syk show that syk substrates are more poorly activated by a non-cross-reacting antigen (fig. 3b). This mechanism provides a type of activation memory, although without knowing the starting point for a given subject's basophils it is not yet possible to exploit this fact to explore the prior activation history of the basophil or mast cell. Parenthetically, figure 3a shows that in the natural state (i.e., no apparent prior downregulation), the relative expression of syk in human basophils is concordant with the relative activation of sig- naling elements that are thought to be substrates of syk [35].

A fascinating attribute of the syk loss phenomenon is its integrative nature. Levels of stimulation that do not result in mediator secretion can nevertheless result in loss of syk, given enough time. In other words, it is possible to treat basophils under full secretory conditions with suboptimal concentrations of antigen and not observe secretion, but continue to observe loss of syk. This observation may have relevance to understanding clinical desensitization regimens where progressive dosing of antigen is used to desensitize a sensitive patient. This attractive hypothesis has yet to be tested, although an in vitro analog has been studied [36-39]. A second attribute of the syk loss phenomenon is that it does not require the activity of syk itself [35] (see fig. 4a). In figure 4a, 3 outcomes are 
examined. Third-generation syk inhibitors inhibit histamine release with an $\mathrm{IC}_{50}$ of approximately $0.3 \mu \mathrm{M}$. In data not shown, many signaling elements downstream of syk activity are also inhibited with an $\mathrm{IC}_{50}$ of approximately $0.3 \mu \mathrm{M}$. However, the loss of syk itself in 24-hour cultures is not inhibited by inhibition of syk activity, except at concentrations 400 -fold higher than the expected $\mathrm{IC}_{50}$. This is true for two third-generation inhibitors tested. In addition, loss or downregulation of the antigenspecific IgE/FceRI (which requires many hours to days) is also not inhibited by syk inhibitors. In others words, like SHIP phosphorylation and recruitment, syk and FceRI loss are only sensitive to src family kinase inhibitors, not syk inhibitors. Transfection of human basophils with the tandem SH2 domain of syk to block the recruitment of wild-type syk to the receptor ITAM does inhibit its loss (fig. 4b). Therefore, syk must be recruited to the receptor to be processed for loss by cbl ligase, but the enzymatic activity of syk is not necessary. Before exploring the significance of this observation further, it is worth exploring one other possible mechanism of nonspecific desensitization.

Studies using rat basophilic leukemia cells noted that aggregation of antigen-specific IgE/receptor resulted in a rapid loss of the receptor from the cell surface [40]. In human basophils and mast cells, receptor loss does not occur in the time frame of cellular desensitization [41]. However, more recent studies have found that over the course of days, cell surface receptor can be lost and a portion of this receptor is degraded (at least in vitro) [42]. The same studies in RBL cells that noted loss of aggregated receptors also noted some loss of nonaggregated receptors [43]. This would provide an alternative mechanism of nonspecific desensitization in human basophils, if observed. There is some loss of noninvolved receptors in human basophils, although it is slow and loss rarely exceeds $10 \%$ of the original density of receptors [44]. As in the case of syk loss and SHIP phosphorylation/recruitment, the loss of receptors is not sensitive to the activities of syk [44].

Three processes that result in downregulation of the IgE-mediated response, receptor loss (very slow), syk loss (slow) and SHIP recruitment (fast) all operate independently of the activities of syk (fig. 4a). This set of observations suggested the hypothesis that all forms of desensitization would operate independently of syk activation. If this were found true, it would also suggest a therapeutic strategy. Recent studies with a third generation syk inhibitor demonstrated that it had no influence on the progress of desensitization in a standard histamine re- lease desensitization assay [44]. In other words, basophils and mast cells could be incubated with stimulus in the absence of extracellular calcium and the presence of syk inhibitor, in this case a Novartis inhibitor, NVP-QAB205, the cells were washed to remove the inhibitor (and stimulus), and rechallenged with stimulus in a calcium-containing buffer (secretion-permissive) to find that they were completely desensitized. Since this assay assesses the entire panoply of downregulatory reactions that follow stimulation, not just the 3 mentioned above, it provides a test of the hypothesis. The conclusion is that no aspect of desensitization is sensitive to the activities of syk. Parenthetically, since there was no effect of having the drug present, there would also be no concerns about the relative selectivity of the drug. Additional studies showed that the syk inhibitor also did not alter the rate of nonspecific desensitization or desensitization that suppresses leukotriene release.

Logically, if desensitization is not affected by a syk inhibitor, it should be possible to include syk inhibitor in a full reaction and not observe secretion but subsequently observe that the cell could no longer be activated. This is indeed the case. Cells stimulated in the presence of extracellular calcium and the presence of syk inhibitor did not secrete. However, when the drug was washed away (and it was demonstrated that washing effectively removed the drug), and the cells restimulated, no secretion occurred. Figure 5 shows this type of experiment using human airway tissue and monitoring the smooth muscle contraction that occurred due to mast cell secretion of histamine and leukotrienes [44]. The tracing designated ' 1 ' shows a normal smooth muscle contraction after stimulation with antigen and ' 3 ' shows that after washing for $60 \mathrm{~min}$ further stimulation was ineffective. In other words, the mast cells secreted but were ultimately desensitized to further stimulation. Tracing ' 2 ' shows that syk inhibitor could be added to the tissue for $90 \mathrm{~min}$, washed away and a normal smooth contraction followed stimulation with antigen. This showed that the drug is effectively washed away. In tracing ' 4 ', drug plus antigen is added to the tissue and there is no contraction and yet when the tissue is washed for $60 \mathrm{~min}$ to remove the drug and stimulus, further stimulation is ineffective (tracing ' 5 '). In other words, the tissue was desensitized without ever secreting. The proposed therapeutic value of this study is that patients undergoing rapid desensitization therapy, which is notoriously responsible for many iatrogenic anaphylactic episodes in the physician's office, could be loaded, one dose only, with a syk inhibitor, treated with antigen and still enter a desensitized state 
Fig. 5. Inducing anergy in mast cells in human lung tissue with syk inhibitor and antigen [abstracted from 44]. After overnight sensitization with ragweed antigen E-specific IgE containing serum, human large airway tissue (2- to 5-mm bronchioles) was hung in a bath to monitor smooth muscle contraction. The tissue was stimulated with ragweed antigen $\mathrm{E}$ under various conditions (see text) and contraction followed over several hours. The NVPQAB205 syk inhibitor was used at $1 \mu \mathrm{M}$.

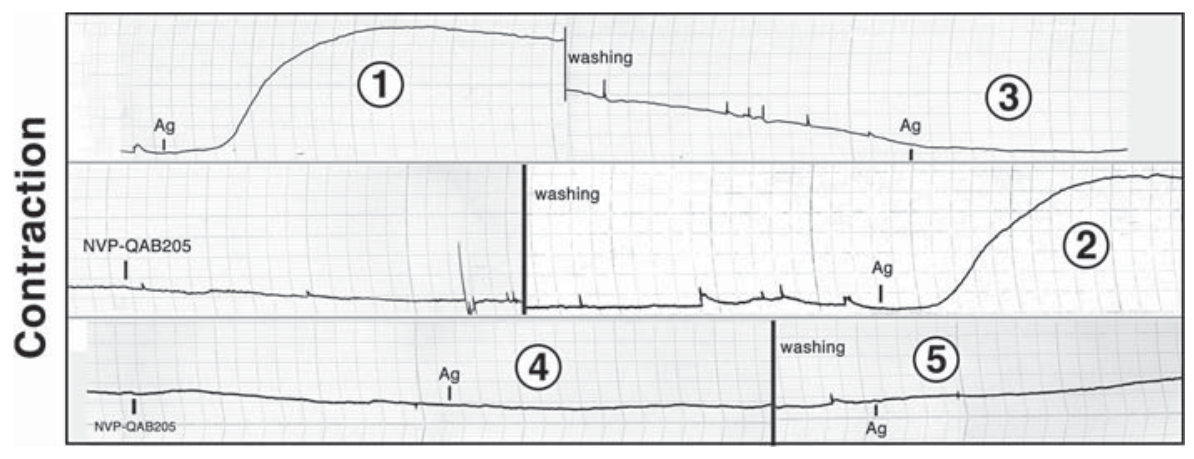

without the risk of anaphylaxis. As the drug was metabolized, remaining antigen would be unable to initiate a mast cell or basophil reaction because the cells would be desensitized without secreting.

The therapeutic potential is questionable because there is great caution in the industry about using syk inhibitors. Syk is expressed in all leukoctyes (even $\alpha \beta \mathrm{T}$ cells to a small extent) and is present in many other cell types. Chronic administration is unpalatable and even a one time dose might require caution. Therefore, it is not clear when or if a syk inhibitor can enter the pharmacy. However, there are related options. The logic of this approach can be applied to elements downstream of syk. Any signaling step that requires syk activity must itself be unnecessary to the process of desensitization. This becomes a powerful tool for exploring what types of signaling steps are required for desensitization, but before exploring this approach, it is useful to note that the first test of the logic was to explore the role for PI3 kinase. In human basophils, the activity of PI3 kinase is dependent on syk activity. For example, a syk inhibitor inhibits the phosphorylation of the regulatory subunit of PI3 kinase and this step is required for recruitment and activity of PI3 kinase. It would be predicted, therefore, that inhibition of PI3 kinase would not block desensitization either. In the same types of experiments described for the syk inhibitor, the PI3 kinase inhibitor, LY294002, did not block desensitization [44]. PI3 kinase is also a ubiquitous enzyme, but the pharmaceutical industry has already generated PI3 kinase inhibitors that are specific and in the clinic. IgE-mediated secretion in human basophils is dependent only on PI $3 \mathrm{~K} \delta$ and this is likely the case for human mast cells. There exist PI3K $\delta$-specific inhibitors and they would serve the same purpose as a syk inhibitor in the physician's office during rush desensitization or immunotherapy.

\section{Further Exploration of Desensitization Mechanisms}

Some coverage of the process of nonspecific desensitization was discussed above. Although these mechanisms also apply to specific desensitization, the signaling studies indicate that SHIP controls events downstream of early signaling. However, stimulation of basophils with antigen results in a transient activation of even the earliest steps in signaling [15]. Similar transience is observed in rodent mast cells $[45,46]$, but the rapid loss of receptor would predict transience of early signaling. Therefore, it is difficult to assess the significance of transience in the rodent model. The data obtained from studies of human basophils support the idea that there is a dominant process of downregulation that occurs prior to the activation of syk and prior to the activation of SHIP. For example, the activation site of lyn kinase is transiently phosphorylated and so too is the phosphorylation of SHIP. Logically, SHIP could not be acting on the earliest steps of activation if it is also transiently active. Some other process is ultimately responsible for returning the cell to its resting state, beginning with the earliest step (but presumably prior, in time, to the very slow eventual loss of receptor which could be the ultimate shut-down of receptor-mediated signaling). The mechanism is currently unknown, but the use of a syk inhibitor has shed some light on which mechanisms, long thought to be viable candidates, are not explanatory. The logic is that described above, if a reaction step requires the activity of syk to become active, then it cannot be relevant to the desensitization process because inhibition of syk does not inhibit desensitization. Three possible mechanisms have been explored.

The polymerization of actin has been proposed as a mechanism of downregulation [47-49]. The underlying proposal is that FceRI signaling occurs exclusively in lipid rafts where the necessary early signaling molecules re- 
side. For example, lyn is thought to localize to the specialized domains of the plasma membrane that are considered lipid rafts. The operating model has been that FceRI resides outside the rafts, separate from lyn, but that aggregation results (by mechanisms unknown) in relocalization of the receptor to the rafts whereupon the reaction can be initiated. The polymerization of actin is a downstream event of activation and by mechanisms unknown, actin has been thought to re-sort the signaling components in the rafts, effectively plucking the receptor out of the rafts to disengage it from the necessary signaling molecules. There was general agreement by a variety of groups that inhibitors of actin polymerization greatly enhanced mediator release from basophils and mast cells of all species [50-53]. This would be a predicted behavior if the actin polymerization model were correct. In studies of RBL cells, inhibition of actin polymerization resulted in prolongation of early signaling, also consistent with the model. However, in human basophils, stimulated actin polymerization is completely inhibited by syk inhibitors [54]. There is a state of resting actin polymerization that is unaffected by syk inhibitors, so it remained possible that resting levels were sufficient to mediate lipid raft reshuffling. However, the inhibitor, latrunculin A, not only inhibits stimulated actin polymerization, but induces the dismantling of all cellular polymerized actin. Treatment of basophils with latrunculin A has no effect on desensitization. Treatment also does not alter the transience of several signaling events in the absence of extracellular calcium, although it does prolong the phosphorylation of syk in the presence of extracellular calcium, but not one of its substrates. Cytochalasin B inhibits stimulated actin polymerization and also has no effect on desensitization or the transience of early signaling with or without extracellular calcium. These results lead to the conclusion that actin polymerization is not the operative mechanism of early desensitization/downregulation.

A related mechanism has been suggested, although it is not easy to visualize precisely what is being proposed to happen. Many years ago it was observed that as the aggregation reaction matures, it becomes more difficult to dissociate a multivalent antigen with its monovalent hapten $[55,56]$. Theoretical considerations predict that as a multivalent antigen makes progressively more contacts (ligand interactions) with cell surface antibody, some slowing in the ability to dissociate the antigen would be expected. However, theoretical predictions indicate that the rate of antigen elution in the presence of excess monovalent hapten would slow by 2 - to 4 -fold [57]. Experimental measurements show a 50 - to 100 -fold slowing. It was proposed that the slower elution rate resulted from immobilization of the receptor. A receptor that could not readily diffuse away from an aggregate after the antigen had been dissociated would likely rebind the antigen, giving the appearance of slower antigen elution. The concept of immobilization was vague, but it was also proposed based on caging models of plasma membranes that immobilization involved the cytoskeleton and possibly polymerized actin [57]. It is relatively straightforward to observe the slowed elution effect in human basophils by sensitizing the cells with a DNP-specific IgE, stimulating the cells with a DNP-GFP conjugate and observing DNPGFP binding by flow cytometry. DNP-GFP is eluted with DNP-lysine. Treatment of the cells during stimulation with DNP-GFP with either latrunculin A, PP1 (a src-family kinase inhibitor) or NVP-QAB205 (a syk inhibitor) or incubation at $4^{\circ} \mathrm{C}$ eliminates the slowing effect [54]. In other words, the process is sensitive to a syk inhibitor, which removes its relevance for desensitization, and it is also sensitive to actin polymerization inhibition, which further removes its relevance to desensitization. Clearly the effect is real and dependent on signaling, but syk and actin dependence suggests that it does not explain the process of desensitization.

The activity of src family kinase, as noted above, is critical to both activation and self-termination. PP1, a src-family kinase inhibitor, inhibits desensitization as readily as it inhibits activation [30]. In recent studies, a negative feedback loop has been identified to operate during immunoreceptor activation $[58,59]$. The kinase, csk, can phosphorylate a tyrosine on src family kinases that results in their inactivation (so-called inhibitory tyrosine that induces a fold in the kinase, so that its $\mathrm{SH} 2$ domain at one end of the molecule binds to the phosphotyrosine at its other end, putting the kinase in a closed, inactive configuration). csk is cytosolic and recruited to the reaction complex by the adaptor protein, cbp/PAG. $\mathrm{cbp} / \mathrm{PAG}$ requires phosphorylation prior to recruiting csk and it has been demonstrated for some cell types that an src family kinase causes cbp/PAG phosphorylation. With such a scheme, there is a tight little feedback loop with an active src family kinase like lyn phosphorylating an adaptor that recruits a kinase to shut off the src family kinase. Depending on the rate in which this little feedback loop is engaged, the impulse activation of the src family kinase would be more or less transient. In human basophils, the phosphorylation of cbp/PAG is dependent on syk activity. This would appear to remove this feedback loop from relevance to desensitization. 
Table 1. Summary of the current perspective for studies in human basophils

\begin{tabular}{|c|c|c|c|c|}
\hline Drug & Target & Endpoint process examined & Inhibits & $\begin{array}{l}\text { Inhibits desensiti- } \\
\text { zation of endpoint }\end{array}$ \\
\hline PP1 & src-family kinase & mediator release & yes & yes \\
\hline NVP-QAB205 & syk & $\begin{array}{l}\text { mediator release } \\
\text { receptor loss } \\
\text { syk loss } \\
\text { SHIP phosphorylation } \\
\text { SHP-1 phosphorylation } \\
\text { cbp/PAG phosphorylation }\end{array}$ & $\begin{array}{l}\text { yes } \\
\text { no } \\
\text { no } \\
\text { no } \\
\text { yes } \\
\text { yes }\end{array}$ & no \\
\hline Bis II & PKC & mediator release & no & no \\
\hline Ro- $81-3220$ & $\mathrm{PKC}$ & mediator release & no & no \\
\hline Latrunculin A & actin & mediator release & enhances & no \\
\hline Cytochalasins & polymerization & 'immobilized' receptor & yes & \\
\hline LY294002 & PI3 kinase & mediator release & yes & no \\
\hline
\end{tabular}

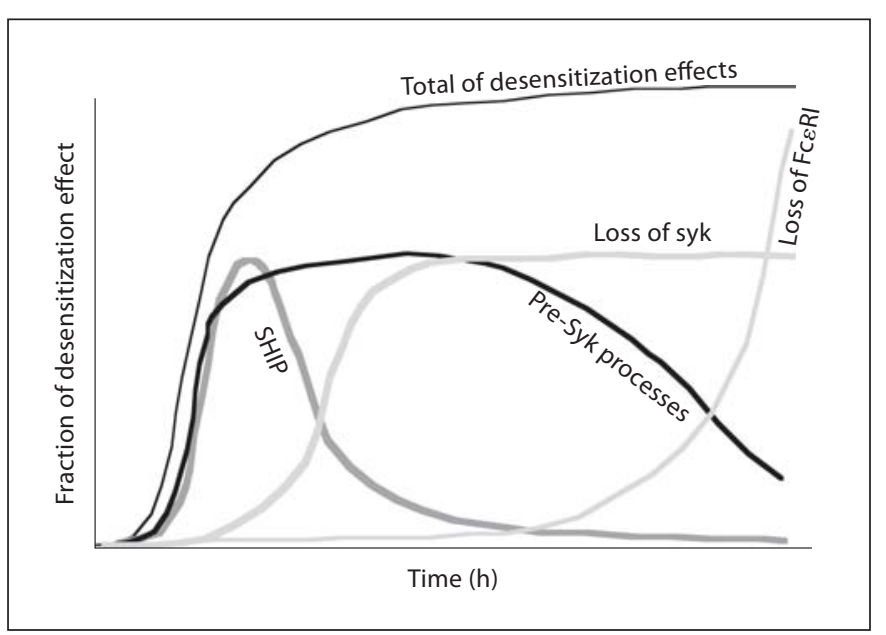

Fig. 6. Relative timing and relative contribution of various downregulatory mechanisms in human basophils. The curves are loose estimates of the relative contributions for 4 known processes involved in downregulation of the IgE-mediated response in human basophils (see text). The abscissa represents a period of about 1 day.

Finally, there are tyrosine phosphatases that are thought to regulate early signaling proteins. Kinases like lyn and syk are dependent on tyrosine phosphorylations for their activity and any dynamically activated tyrosine phosphatases might act to deactivate the early kinases. SHP-1 is one such phosphatase and it has been implicated in IgE-mediated signaling in rodent mast cells [60, 61]. However, in human basophils, phosphorylation of SHP-1 is dependent on syk activity. This characteristic would seem to remove SHP-1 from the list of mechanisms that might explain early self-termination.

The results for cbp/PAG and SHP-1 are less conclusive because specific inhibitors of their activities do not exist and it is, therefore, not yet possible to directly test whether they are irrelevant to desensitization, as was done for PI3 kinase and actin polymerization. The result is highly suggestive but, as yet, inconclusive.

\section{Conclusions}

These results have led to the conclusion that the mechanisms of early desensitization, the process most readily associated with specific desensitization, has not yet been identified. Parenthetically, studies of subthreshold desensitization in murine mast cells indicate that this process is deficient in STAT6-/- mice [39]. It is not clear how a transcription factor would have a direct effect on this particular early downregulatory event, but the interpretation is limited to stating that something about development in a STAT6-negative environment generates a mast cell that loses this downregulatory process.

Figure 6 and table 1 summarize the current perspective for studies in human basophils. If the strength of the stimulus is weak, the dominating process is the unknown early downregulatory step. With stronger stimulation, the effect of SHIP activation becomes a more dominant regulator of ongoing secretion. But this applies only to the early time frame. Further along during activation, progressively more of the deactivation results from the loss of syk. Like the process of SHIP regulation, this has con- 
sequences for both the aggregating stimulus and any non-cross-reacting stimulation that might occur later. Much later in the reaction, loss of the receptor takes place and the cell can, presumably, reset all signaling back to the resting state with aggregated receptor gone from the cell surface. Despite the fact that so many plausible negative feedback loops appear independent of syk activity, and are therefore not responsible for specific desensitization, it seems unlikely that they have no role in regulating secretion. There is good evidence from rodent mast cell models that they do modulate secretion. The question is how this can be true, and yet it can also be true that they play no role in inducing desensitization/anergy in the cells. Modeling the early reaction and coupling it with a heuristic equation to simulate histamine release provides some insight into this question [62]. It can be shown with one of these models that active secretion can be brought up or down with these accessory regulators, such as the cbp/PAG feedback loop, but having them dependent on syk activity does not change the fact that the cell can be desensitized in the presence of a syk inhibitor. In these simple models, it is the rate of desensitization that chang- es in the presence of syk inhibitor, but after $60 \mathrm{~min}$ the cells are still fully desensitized. Whether these models have any relationship to reality seems unlikely, but they do provide some basis for visualizing how the various facts discussed above can be reconciled with one another.

Not surprisingly, there are multiple dynamic homeostatic mechanisms that serve to return the activated mast cell and basophil back to a resting state. Theoretically, variability in the activity of these downregulatory processes can be a means to modulate an individual's specific response. While there are some indirect indications that this perspective is correct, at least in some diseases, no studies in humans have yet proven this point. Nevertheless, it is now apparent that it may be possible to exploit these mechanisms for therapeutic benefit. The observations that a short treatment with a syk inhibitor or a PI3 kinase inhibitor, in conjunction with antigenic stimulation, could render mast cells and basophils anergic are intriguing. If this specific approach is not feasible, it at least demonstrates that there may be related therapeutic approaches.

\section{References}

1 Jouvin MH, Adamczewski M, Numerof R, Letourneur O, Valle A, Kinet JP: Differential control of the tyrosine kinase lyn and syk by the two signaling chains of the high affinity immunoglobulin E receptor. J Biol Chem 1994;269:5918-5925.

2 Kihara H, Siraganian RP: Src homology 2 domains of Syk and Lyn bind to tyrosinephosphorylated subunits of the high affinity IgE receptor. J Biol Chem 1994;269:2242722432.

-3 Yamashita T, Mao SY, Metzger H: Aggregation of the high-affinity IgE receptor and enhanced activity of p53/56lyn protein-tyrosine kinase. Proc Natl Acad Sci USA 1994;91: 11251-11255.

4 Hong H, Kitaura J, Xiao W, Horejsi V, Ra C, Lowell CA, Kawakami Y, Kawakami T: The Src family kinase Hck regulates mast cell activation by suppressing an inhibitory Src family kinase Lyn. Blood 2007;110:25112519.

-5 Xiao W, Nishimoto H, Hong H, Kitaura J, NunomuraS, Maeda-Yamamoto M, Kawakami Y, Lowell CA, Ra C, Kawakami T: Positive and negative regulation of mast cell activation by Lyn via the FcepsilonRI. J Immunol 2005; 175:6885-6892.
6 Odom S, Gomez G, Kovarova M, Furumoto Y, Ryan JJ, Wright HV, Gonzalez-Espinosa C, Hibbs ML, Harder KW, Rivera J: Negative regulation of immunoglobulin E-dependent allergic responses by Lyn kinase. J Exp Med 2004;199:1491-1502.

7 Hernandez-Hansen V, Smith AJ, Surviladze Z, Chigaev A, Mazel T, Kalesnikoff J, Lowell CA, Krystal G, Sklar LA, Wilson BS, Oliver JM: Dysregulated FcepsilonRI signaling and altered Fyn and SHIP activities in Lyndeficient mast cells. J Immunol 2004;173: 100-112.

8 Gibbs BF, Grabbe J: Inhibitors of PI 3-kinase and MEK kinase differentially affect mediator secretion from immunologically activated human basophils. J Leukoc Biol 1999;65: 883-890.

-9 Miura, K, MacGlashan DW Jr: Phosphatidylinositol-3 kinase regulates p21ras activation during IgE-mediated stimulation of human basophils. Blood 2000;96:2199-2205.

10 Miura K, Lavens-Phillips S, MacGlashan DW Jr: Localizing a control region in the pathway to LTC4 secretion following stimulation of human basophils with anti-IgE antibody. J Immunol 2001;167:7027-7037.

11 Lichtenstein LM, De Bernardo R: IgE mediated histamine release: in vitro separation into two phases. Int Arch Allergy Appl Immunol 1971;41:56-71.
12 Sobotka AK, Dembo M, Goldstein B, Lichtenstein LM: Antigen-specific desensitization of human basophils. J Immunol 1979; 122:511-517.

13 MacGlashan DW Jr, Lichtenstein LM: The transition from specific to nonspecific desensitization in human basophils. J Immunol 1981;127:2410-2414.

14 Knol EF, Mul FP, Kuijpers TW, Verhoeven AJ, Roos D: Intracellular events in anti-IgE nonreleasing human basophils. J Allergy Clin Immunol 1992;90:92-103.

15 MacGlashan DW Jr: Two regions of downregulation in the IgE-mediated signaling pathway in human basophils. J Immunol 2003;170:4814-4925.

16 MacGlashan DW Jr, Vilarino N: Nonspecific desensitization, functional memory and the characteristics of SHIP phosphorylation following IgE-mediated stimulation of human basophils. J Immunol 2006;177:1040-1051.

- 17 Kimura T, Sakamoto H, Appella E, Siraganian RP: The negative signaling molecule SH2 domain-containing inositol-polyphosphate 5-phosphatase (SHIP) binds to the tyrosine-phosphorylated beta subunit of the high affinity IgE receptor. J Biol Chem 1997; 272:13991-13996. 
18 Rauh MJ, Kalesnikoff J, Hughes M, Sly L, Lam V, Krystal G: Role of Src homology 2containing-inositol $5^{\prime}$-phosphatase (SHIP) in mast cells and macrophages. Biochem Soc Trans 2003;31:286-291.

-19 Sly LM, Rauh MJ, Kalesnikoff J, Buchse T, Krystal G: SHIP, SHIP2, and PTEN activities are regulated in vivo by modulation of their protein levels: SHIP is up-regulated in macrophages and mast cells by lipopolysaccharide. Exp Hematol 2003;31:1170-1181.

20 Huber M, Helgason CD, Damen JE, Liu L, Humphries RK, Krystal G: The src homology 2-containing inositol phosphatase (SHIP) is the gatekeeper of mast cell degranulation. Proc Natl Acad Sci USA 1998;95:1133011335.

21 Vonakis BM, Gibbons S Jr, Sora R, Langdon JM, MacDonald SM: Src homology 2 domain-containing inositol $5^{\prime}$ phosphatase is negatively associated with histamine release to human recombinant histamine-releasing factor in human basophils. J Allergy Clin Immunol 2001;108:822-831.

-22 Vonakis BM, Vasagar K, Gibbons SP Jr, Gober L, Sterba PM, Chang H, Saini SS: Basophil FcepsilonRI histamine release parallels expression of Src-homology 2-containing inositol phosphatases in chronic idiopathic urticaria. J Allergy Clin Immunol 2007;119:441-448.

23 Gibbs BF, Rathling A, Zillikens D, Huber M, Haas H: Initial Fc epsilon RI-mediated signal strength plays a key role in regulating basophil signaling and deactivation. J Allergy Clin Immunol 2006;118:1060-1067.

24 Magro AM, Alexander A: Histamine release: studies of the inhibitory region of the doseresponse curve. J Immunol 1974;112:17621764.

25 MacGlashan DW Jr, Botana L: Biphasic $\mathrm{Ca}^{++}$ responses in human basophils: evidence that the initial transient elevation associated with mobilization of intracellular calcium is an insufficient signal for degranulation. J Immunol 1993;150:980-991.

26 Dembo M, Goldstein B, Sobotka AK, Lichtenstein LM: Histamine release due to bivalent penicilloyl haptens: control by the number of cross-linked IgE antibodies on the basophil plasma membrane. J Immunol 1978;121:354-358.

-27 Dembo M, Goldstein B, Sobotka AK, Lichtenstein LM: Histamine release due to bivalent penicilloyl haptens: the relation of activation and desensitization of basophils to dynamic aspects of ligand binding to cell surface antibody. J Immunol 1979;122:518528.

28 Damen JE, Liu L, Ware MD, Ermolaeva M, Majerus PW, Krystal G: Multiple forms of the $\mathrm{SH} 2$-containing inositol phosphatase, SHIP, are generated by C-terminal truncation. Blood 1998;92:1199-1205.
29 Leung WH, Bolland S: The inositol 5'-phosphatase SHIP-2 negatively regulates IgE-induced mast cell degranulation and cytokine production. J Immunol 2007;179:95-102.

30 Lavens-Phillips SE, Miura K, MacGlashan DW Jr: Pharmacology of IgE-mediated desensitization of human basophils: effects of protein kinase $\mathrm{C}$ and Src-family kinase inhibitors. Biochem Pharmacol 2000;60:17171727.

-31 MacGlashan D, Miura K: Loss of syk kinase during IgE-mediated stimulation of human basophils. J Allergy Clin Immunol 2004;114: 1317-1324.

32 Youssef LA, Wilson BS, Oliver JM: Proteasome-dependent regulation of Syk tyrosine kinase levels in human basophils. J Allergy Clin Immunol 2002;110:366-373.

33 Paolini R, Molfetta R, Beitz LO, Zhang J, Scharenberg AM, Piccoli M, Frati L, Siraganian R, Santoni A: Activation of Syk tyrosine kinase is required for c-Cbl-mediated ubiquitination of Fcepsilon RI and Syk in RBL cells. J Biol Chem 2002;277:36940-36947.

34 Kepley CL: Antigen-induced reduction in mast cell and basophil functional responses due to reduced Syk protein levels. Int Arch Allergy Immunol 2005;138:29-39.

35 MacGlashan DW Jr: Relationship between Syk and SHIP expression and secretion from human basophils in the general population. J Allergy Clin Immunol 2007;119:626-633.

36 Mendoza GR, Minagawa K: Subthreshold and suboptimal desensitization human basophils. I. Kinetics of decay of releasability. Int Arch Allergy Appl Immunol 1982;68: 101-107.

37 Pruzansky JJ, Patterson R: Desensitization of human basophils with suboptimal concentrations of agonist. Evidence for reversible and irreversible desensitization. Immunology 1988;65:443-447.

38 Komiya A, Hirai K, Iikura M, Nagase H, Yamada H, Miyamasu M, Ohta K, Morita Y, Ra C, Yamamoto K, Yamaguchi M: Induction of basophil desensitization in physiological medium: enhancement after IgE-dependent upregulation of surface IgE binding on basophils. Int Arch Allergy Immunol 2003;130:40-50

39 Morales AR, Shah N, Castells M: AntigenIgE desensitization in signal transducer and activator of transcription 6-deficient mast cells by suboptimal doses of antigen. Ann Allergy Asthma Immunol 2005;94:575580.

40 Isersky C, Rivera J, Segal DM, Triche T: The fate of $\operatorname{IgE}$ bound to rat basophilic leukemia cells. II. Endocy tosis of IgE oligomers and effect on receptor turnover. J Immunol 1983; 131:388-396.

41 MacGlashan DW Jr, Mogowski M, Lichtenstein LM: Studies of antigen binding on human basophils. II. Continued expression of antigen-specific IgE during antigen-induced desensitization. J Immunol 1983;130:23372342 .
42 MacGlashan DW Jr: Endocytosis, re-cycling and degradation of unoccupied FceRI in human basophils. J Leukoc Biol 2007;82:10031010.

43 Furuichi K, Rivera J, Triche T, Isersky C: The fate of IgE bound to rat basophilic leukemia cells. IV. Functional association between the receptors for IgE. J Immunol 1985; 134:17661773.

44 MacGlashan DW Jr, Undem BJ: Inducing an anergic state in mast cells and basophils without secretion. J Allergy Clin Immunol 2008;121:1500-1506.

45 Furuichi K, Rivera J, Isersky C: The fate of $\operatorname{IgE}$ bound to rat basophilic leukemia cells. III. Relationship between antigen-induced endocytosis and serotonin release. J Immunol 1984;133:1513-1520.

46 Molfetta R, Belleudi F, Peruzzi G, Morrone S, Leone L, Dikic I, Piccoli M, Frati L, Torrisi MR, Santoni A, Paolini R: CIN85 regulates the ligand-dependent endocytosis of the IgE receptor: a new molecular mechanism to dampen mast cell function. J Immunol 2005; 175:4208-4216.

47 Pierini L, Harris NT, Holowka D, Baird B: Evidence supporting a role for microfilaments in regulating the coupling between poorly dissociable IgE-Fc epsilonRI aggregates downstream signaling pathways. Biochemistry 1997;36:7447-7456.

- 48 Field KA, Holowka D, Baird B: Compartmentalized activation of the high affinity immunoglobulin $\mathrm{E}$ receptor within membrane domains. J Biol Chem 1997;272:42764280.

49 Holowka D, Gosse JA, Hammond AT, Han X, Sengupta $P$, Smith NL, Wagenknecht-Wiesner A, Wu M, Young RM, Baird B: Lipid segregation and IgE receptor signaling: a decade of progress. Biochim Biophys Acta 2005; 1746:252-259.

50 Urata C, Siraganian RP: Pharmacologic modulation of the IgE or $\mathrm{Ca}^{2+}$ ionophore A23187 mediated $\mathrm{Ca}^{2+}$ influx, phospholipase activation, and histamine release in rat basophilic leukemia cells. Int Arch Allergy Appl Immunol 1985;78:92-100.

- 51 Narasimhan V, Holowka D, Baird B: Microfilaments regulate the rate of exocytosis in rat basophilic leukemia cells. Biochem Biophys Res Commun 1990;171:222-229.

52 Gillespie E, Lichtenstein LM: Histamine from human leukocytes: studies with deuterium oxide, colchicine and cytochalasin B. J Clin Invest 1972;51:2941-2947.

-53 Pfeiffer JR, Seagrave JC, Davis BH, Deanin GG, Oliver JM: Membrane and cytoskeletal changes associated with IgE-mediated serotonin release from rat basophilic leukemia cells. J Cell Biol 1985;101:2145-2155.

54 MacGlashan DW Jr, Vilarino N: Polymerization of actin does not regulate desensitization in human basophils. Submitted. 
-55 Seagrave JC, Deanin GG, Martin JC, Davis $\mathrm{BH}$, Oliver JM: DNP-phycobiliproteins, fluorescent antigens to study dynamic properties of antigen-IgE-receptor complexes on RBL-2H3 rat mast cells. Cytometry 1987;8: 287-295.

-56 MacGlashan DW Jr, Lichtenstein LM: Studies of antigen binding on human basophils. I. Antigen binding and functional consequences. J Immunol 1983;130:2330-2336.

57 Goldstein B, Wofsy C: Why is it so hard to dissociate multivalent antigens from cell surface antibodies? Immunol Today 1996;17: 77-80.

-58 Itoh K, Sakakibara M, Yamasaki S, Takeuchi A, Arase H, Miyazaki M, Nakajima N, Okada M, Saito T: Cutting edge: negative regulation of immune synapse formation by anchoring lipid raft to cytoskeleton through Cbp-EBP50-ERM assembly. J Immunol 2002;168:541-544.
59 Vang T, Abrahamsen H, Myklebust S, Horejsi V, Tasken K: Combined spatial and enzymatic regulation of Csk by CAMP and protein kinase a inhibits T cell receptor signaling. J Biol Chem 2003;278:17597-17600.

60 Pani G, Kozlowski M, Cambier JC, Mills GB Siminovitch KA: Identification of the tyrosine phosphatase PTP1C as a B cell antigen receptor-associated protein involved in the regulation of B cell signaling. J Exp Med 1995;181:2077-2084.

61 Ozawa T, Nakata K, Mizuno K, Yakura H: Negative autoregulation of Src homology region 2-domain-containing phosphatase-1 in rat basophilic leukemia-2H3 cells. Int Immunol 2007;19:1049-1061.
62 Vilarino N, MacGlashan DW Jr: Actin cytoskeleton-dependent down-regulation of early IgE-mediated signaling in human basophils. J Leukoc Biol 2004;75:928-937.

63 Rossi AB, Herlaar E, Braselmann S, Huynh S, Taylor V, Frances R, Issakani SD, Argade A, Singh R, Payan DG, Masuda ES: Identification of the Syk kinase inhibitor R112 by a human mast cell screen. J Allergy Clin Immunol 2006;118:749-755.

64 Vilarino N, MacGlashan D Jr: Transient transfection of human peripheral blood basophils. J Immunol Methods 2005;296:1118.

65 MacGlashan DW Jr, Ishmael S, Macdonald SM, Langdon JM, Arm JP, Sloane DE: Induced loss of Syk in human basophils by non-IgE-dependent stimuli. J Immunol 2008;180:4208-4217. 